\title{
Accidental Decannulation: Systems Thinking, Patient Protection, and Affordable Care
}

The principal conclusion of the 1999 Institute of Medicine report "To Err is Human" is that the major cause of adverse events, rather than being negligent individuals, is actually the result of poorly designed systems. ${ }^{1}$ A major and foundational implication of this and all subsequent Institute of Medicine reports pertaining to healthcare transformation is that healthcare providers (physicians, nurses, therapists, etc) need to work together in teams in a well designed, integrated delivery system to prevent harm. ${ }^{2}$

See the Original Study on Page 2019

\section{Dissection of the Healthcare Delivery Systems}

In this issue, White et al ${ }^{3}$ identify positive impact due to selected practice changes (ie, quality improvement) in the care of tracheotomized patients in a long-term acute care hospital. Healthcare systems are "complex adaptive systems" with many internal components and processes. ${ }^{4,5}$ From a systems viewpoint:

- Patient safety is a function of the (organizational) systems.

- Such systems contain many processes and sub-systems.

- Processes exist within (and among) dynamic system components (Figure).

In optimizing a process such as tracheotomized patients' care, multifactorial interventions are understandably necessary, due to the dynamic complexity of the process within the organizational and patient care framework. The clinical micro-system factors can be dissected into these elements (and sub-elements): patient factors (eg, mental status changes and increased secretions); human component factors (eg, nurses, respiratory therapists, physicians); education factors (eg, knowledge, skills, attitudes, experiences, synthesis skills, and demonstrated competencies/ proficiencies); technical device factors; environmental/ administrative/process factors (eg, staffing ratios, bed-side hand-offs, mandatory hourly rounding, reporting). The clinical micro-system can be influenced by the environment both within and outside the healthcare organization, as

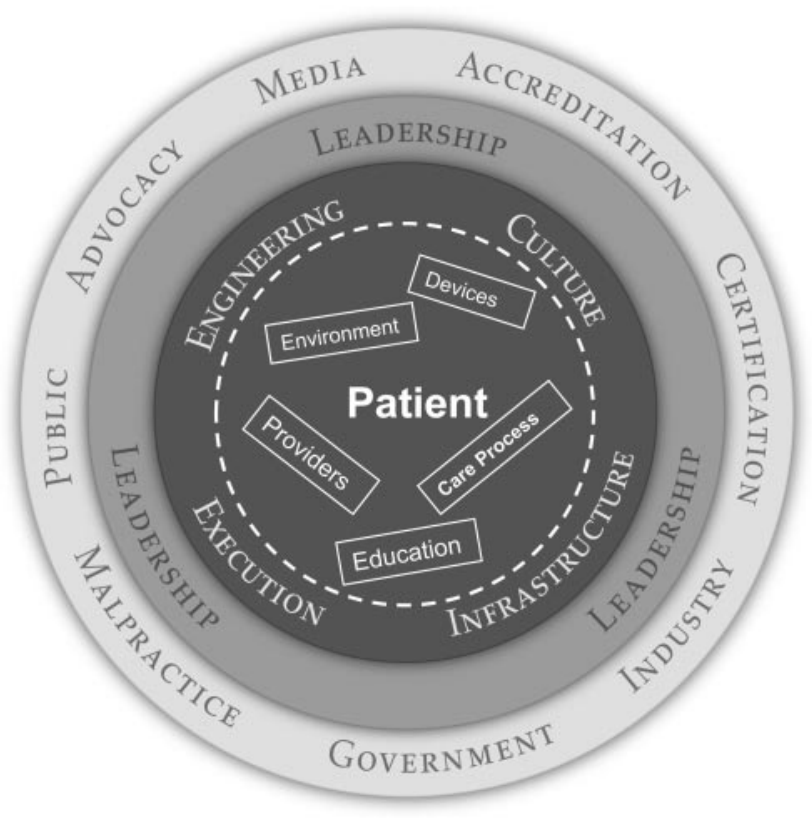

Figure. Integrated healthcare delivery system.

shown. Simulation-based training can be used for healthcare professionals' education, with improved knowledge, skill, behaviors, and patient outcomes. ${ }^{6}$ Safe practicing (eg, in a zero risk healthcare simulation environment) is an increasingly common way to practice safely; simulation helps providers train to demonstrated proficiency standards. ${ }^{7}$ Specialty certification mechanisms can then set requirements for providers in using simulation as a justifiable means to demonstrate competency (eg, Maintenance of Certification in Anesthesiology by the American Board of Anesthesiology). ${ }^{8,9}$

\section{The System in Context}

White et al provide a good example of applied research in quality improvement and the importance of using sound clinical and scientific methods to address patient safety problems. ${ }^{10}$ The authors utilized a systems engineering framework similar to DMAIC (define, measure, analyze, improve, control). ${ }^{11}$ The accidental decannulation problem was defined via prior sentinel event reports (an appraisal of measured institutional adverse event frequency via es- 
tablished benchmarking tools) and root cause analyses. Following these steps, an improvement (intervention) plan was crafted by a multidisciplinary team, which could subsequently be anchored in the culture, and confirmed via ongoing metrics.

We could expand this discussion by moving from the "dance floor" level in operational (tactical) interventions to the conceptual "balcony," with a view of strategic considerations. The difference between a system being described as "complicated" versus "complex" hinges on the capacity for one to predict outcomes by knowing the starting condition ("complicated" model) versus being unable to do so within a "complex" system. In the complex system, the same starting conditions can produce different outcomes due to interactions of intrinsically dynamic system elements. ${ }^{12}$

Humans are intrinsically complex, especially when ill. Complexity has always been associated with healthcare systems. Sir Cyril Chantler stated, "Medicine used to be simple, ineffective and relatively safe. Now it is complex, effective, and potentially dangerous." 13 Because of the intrinsic risks associated with complex healthcare systems, multiple calls to action have occurred via the Institute of Medicine. ${ }^{1,5,14-17}$ These include: creating a culture of safety; creating oversight mechanisms facilitating safest care; education reform; regulatory reform; financial incentives of patient-centric quality care processes based on defining best practices; integrating engineering and healthcare practices; and creating affordable care. As such, systems-based thinking (often not taught within traditional healthcare training curricula ${ }^{18}$ ) offers strategic advantages in approaching clinical deficiencies while facilitating sustainable impact.

\section{Future State}

An unsafe practice is the costliest practice. Consistent with most system changes, quality improvement interventions often require financial investment in the near term. Such organizational infrastructural changes may include improvements in monitoring capacity, dedicated personnel for improved education and quality improvement functions, simulation facilities, etc. Expectations are that the long-term impact of these interventions will be financially justifiable because of improved patient outcomes. As healthcare systems become more patient-centric and accountable, ${ }^{8,19}$ the healthcare industry has unprecedented opportunities to utilize system-based thinking, using information technology enabled, data-driven decision making, and iterative systems engineering approaches to practice improvement. As such, value-based healthcare delivery can be better achieved through multidisciplinary collaboration.
Yue Dong MD

Multidisciplinary Simulation Center

William F Dunn MD

Multidisciplinary Simulation Center

and

Division of Pulmonary and Critical Care Medicine

Department of Medicine

Mayo Clinic

Rochester, Minnesota

\section{REFERENCES}

1. Kohn LT, Corrigan JM, Donaldson MS, editors. To error is human: building a safer health system. Washington, DC: National Academy Press; 1999.

2. Leape L, Berwick D, Clancy C, Conway J, Gluck P, Guest J, et al. Transforming healthcare: a safety imperative. Qual Saf Health Care 2009;18(6):424-428.

3. White AC, Purcell E, Urquhart MB, Joseph B, O'Connor HH. Accidental decannulation following placement of a tracheostomy tube. Respir Care. 2012;57(12):2019-2025.

4. Rouse WB. Health care as a complex adaptive system: implications for design and management. Bridge 2008;38:17-25.

5. Rouse WB, Cortese DA. Engineering the system of healthcare delivery Amsterdam: IOS Press; 2010.

6. Cook DA, Hatala R, Brydges R, Zendejas B, Szostek JH, Wang AT, et al. Technology-enhanced simulation for health professions education: a systematic review and meta-analysis. JAMA 2011;306(9): 978-988.

7. Dunn W, Murphy JG. Simulation: about safety, not fantasy. Chest 2008;133(1):6-9.

8. Boulet JR, Murray DJ. Simulation-based assessment in anesthesiology: requirements for practical implementation. Anesthesiology 2010; 112(4):1041-1052

9. American Board of Anesthesiology. Maintenance of Certification in Anesthesiology (MOCA). http://www.theaba.org/Home/ anesthesiology_maintenance. Accessed September 24, 2012.

10. Batalden PB, Davidoff F. What is "quality improvement" and how can it transform healthcare? Qual Saf Health Care 2007;16(1):2-3.

11. Taner MT, Sezen B, Antony J. An overview of six sigma applications in healthcare industry. Int J Health Care Qual Assur 2007;20: 329-340.

12. Sargut G, McGrath RG. Learning to live with complexity. Harvard Business Review, September 2011. http://hbr.org/2011/09/learningto-live-with-complexity/ar/1. Accessed September 24, 2012.

13. Chantler $\mathrm{C}$. The role and education of doctors in the delivery of health care. Lancet 1999;353(9159):1178-1181.

14. Committee on Quality of Health Care in America; Institute of Medicine. Crossing the quality chasm: a new health system for the 21 st century. Washington DC: National Academies Press; March 2001. http://iom.edu/ Reports/2001/Crossing-the-Quality-Chasm-A-New-Health-System-forthe-21st-Century.aspx. Accessed September 24, 2012.

15. Greiner AC, Knebel E, editors. Health professions education: a bridge to quality. Washington DC: National Academies Press; April 2003. http://www.iom.edu/Reports/2003/Health-Professions-Education-ABridge-to-Quality.aspx. Accessed September 24, 2012.

16. Grossmann C, Goolsby WA, Olsen L, McGinnis JM; Institute of Medicine; National Academy of Engineering. Engineering a learning healthcare system: a look at the future. Workshop summary. Washington DC: National Academies Press; July 2011. http:// 
Accidental Decannulation: Systems Thinking, Patient Protection, and Affordable Care

www.iom.edu/Reports/2011/Engineering-a-Learning-HealthcareSystem.aspx. Accessed September 24, 2012.

The author has disclosed no conflicts of interest.

Correspondence: Yue Dong MD, Multidisciplinary Simulation Center, Mayo Clinic, 200 First Street SW, Rochester MN 55905. E-mail: dong.yue@mayo.edu.

DOI: $10.4187 /$ respcare. 02213
17. Institute of Medicine. Best care at lower cost: the path to continuously learning health care in America. Washington DC: IOM; September 2012. http://www.iom.edu/Reports/2012/Best-Care-atLower-Cost-The-Path-to-Continuously-Learning-Health-Care-inAmerica.aspx. Accessed September 24, 2012.

18. Lucian Leape Institute; National Patient Safety Foundation. Unmet needs: teaching physicians to provide safe patient care. Boston: NPSF; 2010. http://www.npsf.org/wp-content/uploads/2011/10/LLI-UnmetNeeds-Report.pdf. Accessed September 24, 2012.

19. Berwick DM. What 'patient-centered' should mean: confessions of an extremist. Health Aff (Millwood) 2009;28(4):w555-w565. 